A. M. Romaniv ${ }^{1}$, B. V. Zabavsky², S. I. Bilavska²

\title{
ADEQUATE PROPERTIES OF THE ELEMENTS WITH ALMOST STABLE RANGE 1 OF A COMMUTATIVE ELEMENTARY DIVISOR DOMAIN
}

(In boring memory of V.I. Andriychuk on the 70 th anniversary of his birth)

It is shown that in a commutative elementary divisor domain which is not a ring of stable range 1 exist nonzero and nonunit elements with almost stable range 1.

The problem of diagonalization of matrices is a classic one. Specific role in modern research on elementary divisor rings is played by a $K$ - theoretical invariant as the stable range [4]. Important role in studying of the elementary divisor rings played a Hermite rings, i.e. ring in which $1 \times 2$ and $2 \times 1$ matrices over this ring have diagonal reduction. Note that any Hermite ring is a Bezout ring i.e. a ring in which any finitely generated ideal is principal. We have the following result.

Theorem 1[4]. A commutative Bezout ring is Hermite ring if and only if it is a ring of stable range 2 .

Recall, that a ring $R$ is a ring of stable range 2 if for any elements $a, b, c \in R$ the equality $a R+b R+c R=R$ implies that there are some elements $\lambda, \mu$ such that

$$
(a+c \lambda) R+(b+q) R=R .
$$

Recall, that a ring $R$ is a ring of stable range 1 if for any elements $a, b \in R$ the equality $a R+b R=R$ implies that there are some element $t$ such that $(a t+b) R=R$.

Let $\mathrm{R}$ - commutative elementary divisor domain which is not a ring of stable range 1.

By [2] there exists nonzero and nonunit element $a \in R$ with almost stable range 1 (i.e. for any elements $b, c \in R$ such that $a R+b R+c R=R$ exists element $t$ that $a R+(b t+c) R=R)$. In this paper we describe algebraic properties these element $t \in R$.

By [2] we have that the problem "is every commutative Bezout domain an elementary divisor ring" is equivalent to the problem does every commutative Bezout domain contain a nonunit element with almost stable range 1 . In this article gives a more precise description of this elements.

All rings considered will be commutative and have identity. Element $a \in R$ of a commutative ring is called a neat element if for any elements $b, c \in R$ such that $b R+c R+a R=R$ we have $a=r s$ where $r R+b R=R$, $S R+C R=R, r R+s R=R$.

Theorem 2[3]. Let $R$ be a commutative Bezout domain. An element $a$ is a neat element if the factor-ring $R / a R$ is a clean ring.

Recall that a ring is called clean if each element is the sum of the unit and an idempotent.

A commutative ring $R$ is said to be a ring of neat range 1 if for any $a, b \in R$ such that $a R+b R=R$ there exists $t \in R$ such that $a+b t \in R$ is $a$ neat element. We have a next result.

Theorem 3[3]. A commutative Bezout domain is an elementary divisor ring if it is a ring of neat range 1 .

Recall that a commutative ring $\mathrm{R}$ is called an elementary divisor ring if every matrix $A$ over $\mathrm{R}$ admits diagonal reduction, that is there exist

ISSN 1810-3022. Прикл. проблеми мех. і мат. - 2018. - Вип. 16. - С. 33- 35. 
invertible matrices $P$ and $Q$ such that $P A Q$ is a diagonal matrix, $\left(d_{i}\right)$ for which $d_{i}$ is a divisor of $d_{i+1}$.

Recall that a ring $R$ is an exchange ring if for any element $a \in R$ there exists an idempotent e such that $e \in a R$ and $1-e \in(1-a) R$ [13].

$A$ ring $R$ is a ring of idempotent stable range 1 if the condition $a R+b R=R$ for all elements $a, b \in R$ implies that there exists an idempotent $e \in R$ such that $a+$ be is an invertible element of the ring $R$.

We have the following result.

Theorem 4[1]. Let $\mathrm{R}$ be a commutative ring. The following properties are equivalent:

1) $R$ is an exchange ring,

2) $R$ is a clean ring,

3) $\mathrm{R}$ is a ring of idempotent stable range 1 .

Proposition. Let $R$ be a commutative ring. Nonzero element $a \in R$ is element with almost stable range 1 if and only if a factor-ring $R / a R$ is a ring of stable range 1 .

P r o o f. Denote $\bar{R}=R / a R$ and $\bar{b}=b+a R, \bar{c}=c+a R$. If $\bar{R}$ is a ring of stable range 1 and $\bar{b} \bar{R}+\bar{c} \bar{R}=\bar{R}$, then exists element $\bar{t} \in R$ such that $(\bar{b} \bar{t}+\bar{c}) \bar{R}=\bar{R}$. Since $\bar{R}=R / a R$ and by [1] we have $a R+(b t+c) R=R$, where $\overline{\mathrm{t}}=t+a R$, i.e. $a$ is element with almost stable range 1 . We notice, that condition $\mathrm{aR}+(\mathrm{bt}+\mathrm{c}) \mathrm{R}=\mathrm{R}$ implies $\overline{\mathrm{b} R}+\overline{\mathrm{CR}}=\overline{\mathrm{R}}$. Proposition is proved.

Nonzero element $a$ of a commutative ring $R$ is said to be adequate to the element $b \in R \quad(a A b$ denote this fact) if we can find such elements $r, s \in R$ that the decomposition $\mathrm{a}=\mathrm{rs}$ satisfying the following properties:

1) $r R+b R=R$,

2) $s^{\prime} R+b R \neq R$ for any noninvertible divisor $s^{\prime}$ of element $s$.

If for any element $b \in R$ we have $a A b$ then we say that element $a$ is adequate. If any nonzero element of a ring $R$ is an adequate element then $R$ is called an adequate ring. An addition we notice simple fact: for any nonzero element $\mathrm{a}$ of $\mathrm{R}$ we have $\mathrm{aAa}$. The most obvious examples of adequate elements are units, square free elements and factorial elements [4]. By [2] we have that an adequate element is a neat element.

The main result of this paper is a next Theorem.

Theorem 5. Let $\mathrm{R}$ be a commutative elementary divisor domain, which is not a ring of stable range 1 . Then there exists nonunit and nonzero element $a \in R$ and for any $b, c \in R$ such that $a R+b R+c R=R$ there exists element $\mathrm{t} \in \mathrm{R}$ such that $\mathrm{aR}+(\mathrm{bt}+\mathrm{c}) \mathrm{R}=\mathrm{R}$ and $\mathrm{aAt}$.

$\mathrm{P} r$ o o f. Let $\mathrm{R}$ be a commutative elementary divisor domain. By [4] $\mathrm{R}$ is a Bezout domain. By Theorem $3 \mathrm{R}$ is a ring of a neat range 1 . Since $R$ is not a ring of stable range 1 , then in $R$ exists nonzero and nonunit neat element $a$. By Theorem 2 we have that $\bar{R}=R / a R$ is a clean ring. By Theorem 3 , we have that $\bar{R}=R / a R$ is a ring idempotent stable range 1 . Let $\bar{b}=b+a R, \bar{c}=c+a R$. Then we have if $\bar{b} \bar{R}+\bar{c} \bar{R}=\bar{R}$ there exists idempotent $\overline{\mathrm{e}}=\mathrm{e}+\mathrm{aR}$ such that $\overline{\mathrm{be}}+\overline{\mathrm{c}}$ is an invertible element of $\overline{\mathrm{R}}$. By Proposition we have that $a R+(b e+c) R=R$.

Let $\quad a R \neq e R=d R$, then $a=d a_{0}, \quad e=d e_{0} \quad$ and $\quad a_{0} R+e_{0} R=R \quad$ i.e. $a_{0} u+e_{0} v=1$ for some elements $d, a_{0}, e_{0}, u, v \in R$. Since $\bar{e}^{-2}=\bar{e}$ we have $\mathrm{e}-\mathrm{e}^{2}=$ at for some element $\mathrm{t} \in \mathrm{R}$. Then $\mathrm{e}(1-\mathrm{e})=\mathrm{de}_{0}(1-\mathrm{e})=d a_{0} \mathrm{t}$ and since 
$d \neq 0$ we have $e_{0}(1-e)=a_{0} t$. Since $a_{0} u+e_{0} v=1$ we have

$$
1-e=e_{0}(1-e) u+a_{0}(1-e) v=a_{0} t u+a_{0}(1-e) v=a_{0}(t u+(1-e) v),
$$

i.e. we have $1-e=a_{0} k$, where $k=t u+(1-e) v$. So we proved that $a=a_{0} d$ where $a_{0} R+e R=R$ and $e R \subset d R$ i.e. we have aAe where $r=a_{0}, s=d$ according to the definition of the condition of the adequate of element $a$ to the element e. Theorem is proved.

Corollary. Commutative elementary divisor domain $\mathrm{R}$ which is not a ring of stable range 1 exist nonzero and nonunit elements with almost stable range 1 .

$\mathrm{P} r$ o o f. By Theorem 5 there exists nonunit and nonzero element $a \in R$ and for any $b, c \in R$ such that $a R+b R+c R=R$ there exists element $t \in R$ such that $a R+(b t+c) R=R$. By Proposition $a$ is element with almost stable range 1.

We will notice in the ring of stable range 1 any nonzero and nonunit element is an element with almost stable range 1[2].0

Recall, that a commutative Bezout rings stable range 1 is an elementary divisor rings [3]. Note, that a commutative J -Noetherian Bezout domain which is not a ring of stable range 1 always contain nonzero and nonunit element with almost stable range 1 which is adequate element of this ring.

1. Zabavsky B.V., Bilavska S.I. Every zero adequate rings is an exchange ring // J ournal of Mathematical Sciences. - 2012. - 187, № 2. - P. 153- 156.

2. Zabavsky B. Conditions for stable range of an elementary divisor rings. // Communications in Algebra. - 2016. - 45, № 9 - P. 4062- 4066.

3. Zabavsky B.V. Diagonal reduction of matrices over finite stable range rings. // Matematychni Studii. - 2014. - 41, № 1. - P. 101- 108.

4. Zabavsky B.V. Diagonal reduction of matrices over rings. // Mathematical Studies, Monograph Series, V, XVI, VNTL Pulishers, Lviv. - 2012. - 252 p.

\section{АДЕКВАТНЫЕ СВОЙСТВА ЭЛЕМЕНТОВ ПОЧТИ СТАБИЛЬНОГО РАНГА 1 КОММУТАТИВНОЙ} ОБЛАСТИ ЭЛЕМЕНТАРНЫХ ДЕЛИТЕЛЕЙ

Показано, что в коммутативной области әлементарных делителей, которая не является кольиом стабильного ранга 1, существуют ненулевые и необратимые әлементы почти стабильного ранга 1.

\section{АДЕКВАТНІ ВЛАСТИВОСТІ ЕЛЕМЕНТІВ МАЙЖЕ СТАБІЛЬНОГО РАНГУ 1 КОМУТАТИВНОЇ} ОБЛАСТІ ЕЛЕМЕНТАРНИХ ДІЛЬНИКІВ.

Показано, що в комутативній області елементарних дільників, яка не є кільием стабільного рангу 1, існують ненульові та необоротні елементи майже стабільного рангу 1.

1Pidstryhach Institute of Applied Problems of Mechanics and Mathematics

2 Ivan Franko National University of Lviv, Lviv 